\title{
Multi-State Dependent Impulsive Control for Holling I Predator-Prey Model
}

\author{
Huidong Cheng, Fang Wang, and Tongqian Zhang \\ College of Science, Shandong University of Science and Technology, Qingdao 266510, China \\ Correspondence should be addressed to Huidong Cheng, chd900517@sdust.edu.cn
}

Received 18 February 2012; Accepted 10 April 2012

Academic Editor: Recai Kilic

Copyright (C) 2012 Huidong Cheng et al. This is an open access article distributed under the Creative Commons Attribution License, which permits unrestricted use, distribution, and reproduction in any medium, provided the original work is properly cited.

According to the different effects of biological and chemical control, we propose a model for Holling I functional response predator-prey system concerning pest control which adopts different control methods at different thresholds. By using differential equation geometry theory and the method of successor functions, we prove that the existence of order one periodic solution of such system and the attractiveness of the order one periodic solution by sequence convergence rules and qualitative analysis. Numerical simulations are carried out to illustrate the feasibility of our main results which show that our method used in this paper is more efficient and easier than the existing ones for proving the existence of order one periodic solution.

\section{Introduction}

More and more scholars have paid close attention, and studied impulsive differential equation since the 1980s. Impulsive differential equation theory, especially the one in a fixed time, has been deeply developed and widely applied in various fields through years of research [1-5]. In population dynamical system, Tang et al. [6] discussed the stage-structure system for single population with birth pulse and got the existence and stability of periodic solution; Liu et al. [7-9] studied the impulse control strategy of Lotka-Volterra system; he also set up and discussed the Holling type II predator-prey model with impulse control strategy. Ballinger and Liu [10] discussed the persistence of population model with impulse effect. Tang and Cheke [11] first proposed the "Volterra" model with state-dependence, and they applied this model to pest management and proved existence and stability of periodic solution of first and second orders. Then, Liu et al. [12] also proposed bait-dependent digestive model with state pulse, and the model had the existence of positive periodic solution and stability of orbit. Recently, Jiang and Liu et al. [12-14] have proposed pest 
management model with state pulse and phase structure and several predator-prey models with state pulse and had the existence of semitrivial periodic solution and positive periodic solution and stability of orbit.

In consideration of predator-prey capacity, Holling [15] proposes three different predations with functional response based on experiments; the average predator-prey system with Holling response is as follows:

$$
\begin{aligned}
& x^{\prime}(t)=x g(x)-y \phi(x), \\
& y^{\prime}(t)=-d y+\operatorname{ey} \phi(x),
\end{aligned}
$$

where $x$ represents the prey's density, while $y$ is the predator's; $g(x)$ is the unit rate of prey density in lack of predators; $\phi(x)$ is the Holling functional response, among which Holling type I functional response is

$$
\phi(x)= \begin{cases}c x, & x \leq x_{0} \\ c x_{0}, & x>x_{0}\end{cases}
$$

where $c$ is a constant; when the amount of prey is greater than certain threshold value $x_{0}$, predatory rate is a constant. Referring to [15] for details.

As the Lotka-Volterra predator-prey system with Holling functional response is more practical, many authors have studied it [12, 14, 15]. The researches mostly focus on Lotka-Volterra predator-prey model with Holling type II or Holling type III functional response in contrast to the model with Holling type I. This paper sets up a state-dependent impulsive mathematical model concerning pest control which adopts different control methods at different thresholds and adopts new mathematic method to study existence and attractiveness of order one periodic solution of such system; thus the following pest-control model with Holling type-I functional response is set up:

$$
\begin{aligned}
x^{\prime}(t)=r x(t)-c x(t) y(t), & \\
y^{\prime}(t)=-d y(t)+e c x(t) y(t), & \\
x^{\prime}(t)=r x(t)-c x_{0} y(t), & x \neq x_{0}, \\
y^{\prime}(t)=-d y(t)+e c x_{0} y(t), & \quad x>x_{0}, \\
\Delta x(t) & =0, \quad x=h_{2}, \quad y \leq y^{*}, \\
\Delta y(t) & =\delta, \quad x=h_{1}, \quad y>y^{*}, \\
\Delta x(t) & =-\alpha x(t), \quad x=h_{2}, \\
\Delta y(t) & =-\beta y(t)+q, \quad
\end{aligned}
$$

where $r, c, d, e, h_{1}$, and $h_{2}$ are all positive constants, $x(t)$ and $y(t)$ represent the densities of prey (pest) and predator (natural enemy), respectively; $r$ is the intrinsic growth rate of the prey; $d$ denotes the death rate of the predator; $(\alpha, \beta) \in(0,1)$ represent the proportion of killed prey and predator by spraying pesticides respectively, $\delta>0$ is the number of natural enemies released at this time $t_{h_{1}}$, when the amount of the prey reaches the threshold $h_{1}$ at time $t_{h_{1}}$, control measures are taken (releasing natural enemies) and the amount of predator abruptly turns to $y\left(t_{h_{1}}\right)+\delta$. When the amount of prey reaches the threshold $h_{2}$ at time $t_{h_{2}}$, control measures are taken and the amount of prey and predator abruptly turns to $(1-\alpha) h_{2}$ and 
$(1-\beta) y\left(t_{h_{2}}\right)$, respectively. $\Delta x=x\left(t^{+}\right)-x(t), \Delta y=y\left(t^{+}\right)-y(t), x\left(t^{+}\right)=\lim _{w \rightarrow 0} x(t+w), y\left(t^{+}\right)=$ $\lim _{w \rightarrow 0} y(t+w)$. Referring to [12] for details.

\section{Preliminaries}

We first consider the model (1.3) without impulse effects:

$$
\begin{aligned}
& x^{\prime}(t)=r x(t)-c x(t) y(t), \quad \\
& y^{\prime}(t)=-d y(t)+e c x(t) y(t), \quad x \leq x_{0}, \\
& x^{\prime}(t)=r x(t)-c x_{0} y(t), \quad x>x_{0} . \\
& y^{\prime}(t)=-d y(t)+e c x_{0} y(t) .
\end{aligned}
$$

We consider the following function:

$$
V(x, y)=\int_{x^{*}}^{x} \frac{-d+e \phi(s)}{\phi(s)} d s+\int_{y^{*}}^{y} \frac{s-y^{*}}{s} d s,
$$

we can easily know that $V(x, y)$ is positive definite in the first quartile and fits for all conditions of Lyapunov function.

We can get that

$$
V^{\prime}(x, y)=\frac{e x y^{*}}{\phi(x)}\left(\phi(x)-\phi\left(x^{*}\right)\right)\left(\frac{\phi\left(x^{*}\right)}{x^{*}}-\frac{\phi(x)}{x}\right) .
$$

It is easily proved that $V^{\prime}(x, y) \equiv 0$ on condition that $x \leq x_{0}$; so all solutions of model (1.3) form a set $\left\{(x, y) / V(x, y) \leq V\left(x_{0}, y^{*}\right)\right\}$ are closed trajectory $V(x, y)=C$, where $0<C<$ $V\left(x_{0}, y^{*}\right)$.

Since $V^{\prime}(x, y)>0$ on condition that $x>x_{0}$; so the trajectory of system (2.1) passes through closed curve $V(x, y)=C$ when it is out of the curve $V(x, y)=V\left(x_{0}, y^{*}\right)$.

Therefore, we observe the straight line:

$$
L(x, y)=y+x-n, \quad n>0, x_{0}<x \leq h .
$$

The derivative of $L(x, y)$ along (2.1) is that

$$
\begin{aligned}
L^{\prime}(x, y) / L=0 & =x^{\prime}+y^{\prime}=-d y+e c x_{0} y+r x-e c x_{0} \\
& =-\left(d n-e c x_{0} n+c x_{0} n+c x_{0} h-d x_{0}\right)-\left(e c x_{0}-r-c x_{0}\right) \\
& \leq d h-e c x_{0}^{2}+r h+c x_{0} h-\left(d-e c x_{0}+c x_{0}\right) n
\end{aligned}
$$

We have that $L^{\prime} / L=0<0$ on condition that $n>\left(\left(d h-e c x_{0}^{2}+r h+c x_{0} h\right) /\left(d-e c x_{0}+c x_{0}\right)\right)$. Therefore, we can get the following Lemma.

Lemma 2.1. The system (2.1) possesses: 


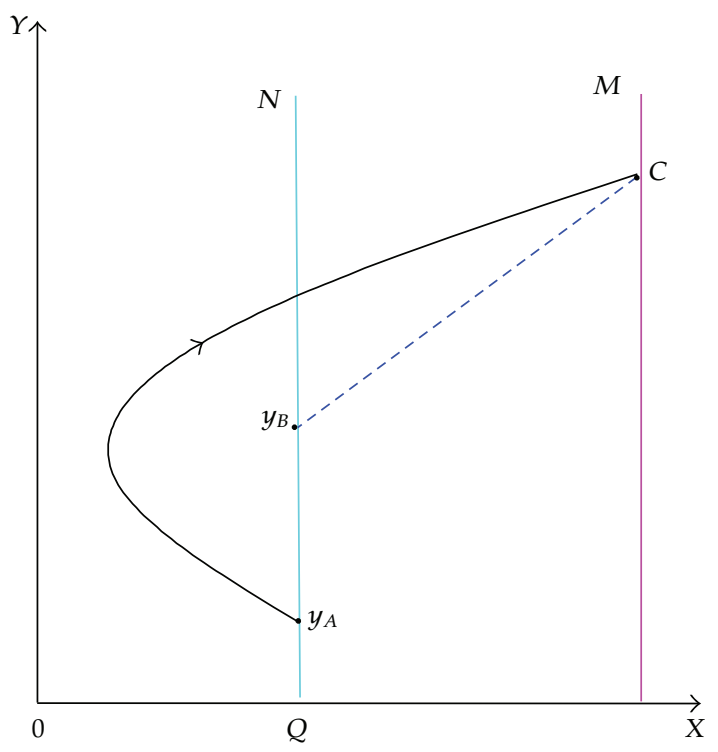

Figure 1

(I) two steady states $0(0,0)$ - saddle point, and $R(d / e c, r / c)=R\left(x^{*}, y^{*}\right)$-stable centre on the condition that $x \leq x_{0}$ and that $d \leq e c x_{0}$;

(II) the trajectory of system (2.1) goes across the straight line $y+x-n=0$ from the right to the left on condition that $x_{0} \leq x \leq h$ and that $n>\left(\left(d h-e c x_{0}^{2}+r h+c x_{0} h\right) /\left(d-e c x_{0}+c x_{0}\right)\right)$ and intersects with the straight line $x=x_{0}$.

Definition 2.2. Suppose that the impulse set $M$ and the phase set $N$ are both lines, as shown in Figure 1. Define the coordinate in the phase set $N$ as follows: denote the point of intersection $Q$ between $N$ and $x$-axis as $O$, then the coordinate of any point $A$ in $N$ is defined as the distance between $A$ and $Q$ and is denoted by $y_{A}$. Let $C$ denote the point of intersection between the trajectory starting from $A$ and the impulse set $M$, and let $B$ denote the phase point of $C$ after impulse with coordinate $y_{B}$. Then, we define $B$ as the successor point of $A$, and then the successor function [16] of point $A$ is that $f(A)=y_{B}-y_{A}$.

Lemma 2.3. In system (1.3), if there exist $A \in N, B \in N$ satisfying successor function $f(A) f(B)<$ 0 , then there must exist a point $P(P \in N)$ satisfying $f(P)=0$ the function between the point of $A$ and the point of $B$, thus there is an order one periodic solution in system (1.3).

In this paper, we assume that the condition $d \leq e c x_{0}$ holds. By the biological background of system (1.3), we only consider $D=\{(x, y): x \geq 0, y \geq 0\}$.

This paper is organized as follows. In the next section, we present some basic definitions and important lemmas as preliminaries. In Section 3, we prove the existence for an order one periodic solution of system (1.3). The sufficient conditions for the attractiveness of order one periodic solutions of system (1.3) are obtained in Section 4. At last, we state conclusion, and the main results are carried out to illustrate the feasibility by numerical simulations. 


\section{Existence of Order One Periodic Solution}

In this section, we shall investigate the existence of an order one periodic solution of system

(1.3) by using the successor function defined in this paper. For this goal, we denote

$$
\begin{gathered}
M_{1}=\left\{\frac{(x, y)}{x}=h_{1}, 0 \leq y \leq \frac{r}{c}+\delta\right\}, \\
M_{2}=\left\{(x, y) \mid x=h_{2}, y \geq 0\right\}, \\
N_{1}=I\left(M_{1}\right)=\left\{(x, y) \mid x=h_{1}, \frac{r}{c}<y \leq \frac{r}{c}+\delta\right\}, \\
N_{2}=I\left(M_{2}\right)=\left\{(x, y) \mid x=(1-\alpha) h_{2}, y \geq 0\right\} .
\end{gathered}
$$

Isoclinic line is denoted respectively by lines:

$$
\begin{aligned}
& L_{1}=\left\{(x, y) \mid y=\frac{r}{c}, 0 \leq x \leq x_{0}\right\}, \\
& L_{2}=\left\{(x, y) \mid x=\frac{d}{e c}, 0 \leq x \leq x_{0}, y \geq 0\right\}, \\
& L_{3}=\left\{(x, y) \mid y=\frac{r}{c x_{0}} x, x>x_{0}, y \geq \frac{r}{c}\right\} .
\end{aligned}
$$

For the convenience, if $P \in \Omega-M, F(P)$ is defined as the first point of intersection of $C^{+}(P)$ and $M$, that is, there exists a $t_{1} \in R_{+}$such that $F(P)=\Pi\left(P, t_{1}\right) \in M$, and for $0<t<t_{1}, \Pi(P, t) \notin M$; if $B \in N, R(B)$ is defined as the first point of intersection of $C^{-}(P)$ and $N$, that is, there exists a $t_{2} \in R_{+}$such that $R(B)=\Pi\left(B,-t_{2}\right) \in N$, and for $-t<t<0, \Pi(B, t) \notin N$.

For any point $P$, denote $y_{P}$ as its ordinate. If the point $P\left(h, y_{P}\right) \in M$, pulse occurs at the point $P$, the impulsive function transfers the point $P$ into $P^{+} \in N$. Without loss of generality, we assume the initial point of the trajectory lies in phase set $N$ unless otherwise specified.

According to the practical significance, in this paper we assume that the set $N$ always lies in the left side of stable centre $R$, that is, $h_{1}<r / c,(1-\alpha) h_{2}<r / c$.

In the light of the different position of the set $N_{1}$ and the set $N_{2}$, we consider the following three cases.

Case $1\left(0<h_{1}<d / e c\right)$. In this case, set $M_{1}$ and $N_{1}$ are both in the left side of stable center $R$ (as shown in Figure 2). Take a point $B\left(h_{1}, r / c+\varepsilon\right) \in N_{1}$ above $A$, where $\varepsilon>0$ is small enough, then there must exist a trajectory passing through $B$ which intersects with the set $M_{1}$ at point $P_{1}\left(h_{1}, y_{p_{1}}\right)$, we have $y_{p_{1}}<r / c$. Since $p_{1} \in M_{1}$, pulse occurs at the point $P_{1}$, the impulsive function transfers the point $P_{1}$ into $P_{1}^{+}\left(h_{1}, y_{p_{1}}+\delta\right)$ and $P_{1}^{+}$must lie above $B$; therefore, inequation $a / b+\varepsilon<y_{p_{1}}+\delta$ holds, thus the successor function of $B$ is that $f(B)=$ $y_{p_{1}}+\delta-(r / c+\varepsilon)>0$.

On the other hand, the trajectory with the initial point $P_{1}^{+}$intersects with $M_{1}$ at point $P_{2}\left(h_{1}, y_{p_{2}}\right)$, in view of vector field and disjointness of any two trajectories, we know 


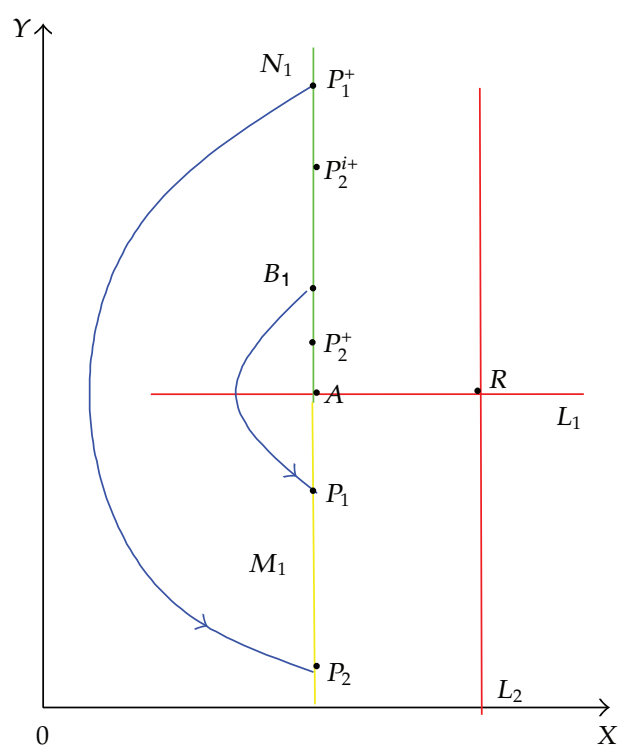

Figure 2

$y_{p_{2}}<y_{p_{1}}<r / c$. Supposing that the point $P_{2}$ is subject to impulsive effects to point $P_{2}^{+}\left(h_{1}, y_{p_{2}^{+}}\right)$, where $y_{p_{2}^{+}}=y_{p_{2}}+\delta$, the position of $P_{2}^{+}$has the following two cases.

Subcase $1.1\left(r / c<y_{p_{2}}+\delta<y_{p_{1}}+\delta\right)$. In this case, the point $P_{2}^{+}$lies above the point $A$ and under $P_{1}^{+}$, we have $f\left(P_{1}^{+}\right)=y_{p_{2}}+\delta-\left(y_{p_{1}}+\delta\right)<0$.

By Lemma 2.3, there exists an order one periodic solution of system (1.3), whose initial point is between $B$ and $P_{1}^{+}$in set $N_{1}$.

Subcase $1.2\left(r / c \geq y_{p_{2}}+\delta\right.$ (as shown in Figure 2)). The point $P_{2}^{+}$lies below the point $A$, that is, $P_{2}^{+} \in M_{1}$, then pulse occurs at the point $P_{2}^{+}$, the impulsive function transfers the point $P_{2}^{+}$ into $P_{2}^{++}\left(h_{1}, y_{p_{2}}+2 \delta\right)$.

If $r / c<y_{p_{2}}+2 \delta<y_{p_{1}}+\delta$, like the analysis of Subcase 1.1, there exists an order one periodic solution of system (1.3).

If $r / c>y_{p_{2}}+2 \delta$, that is, $P_{2}^{++} \in M_{1}$; we repeat the above process until there exists $k \in Z_{+}$such that $P_{2}^{++}$jumps to $P_{2}^{i+}\left(\left(h_{1}, y_{p_{2}}+(k+2) \delta\right)\right.$ after $k$ times' impulsive effects which satisfies $r / c<y_{p_{2}}+(k+2) \delta<y_{p_{1}}+\delta$. Like the analysis of Subcase 1.1, there exists an order one periodic solution of system (1.3).

Now we can summarize the above results in the following theorem.

Theorem 3.1. If $d<e c, 0<h_{1}<d / e c$, then there exists an order one periodic solution of the system (1.3).

Remark 3.2. It shows from the proved process of Theorem 3.1 that the number of natural enemies should be selected appropriately, which aims to reduce releasing impulsive times to save manpower and resources.

Case $2\left(h_{2}<d / e c\right)$. In this case, sets $M_{2}$ and $N_{2}$ are both in the left side of stable center $R$, in the light of the different position of the set $N_{2}$, we consider the following two cases. 


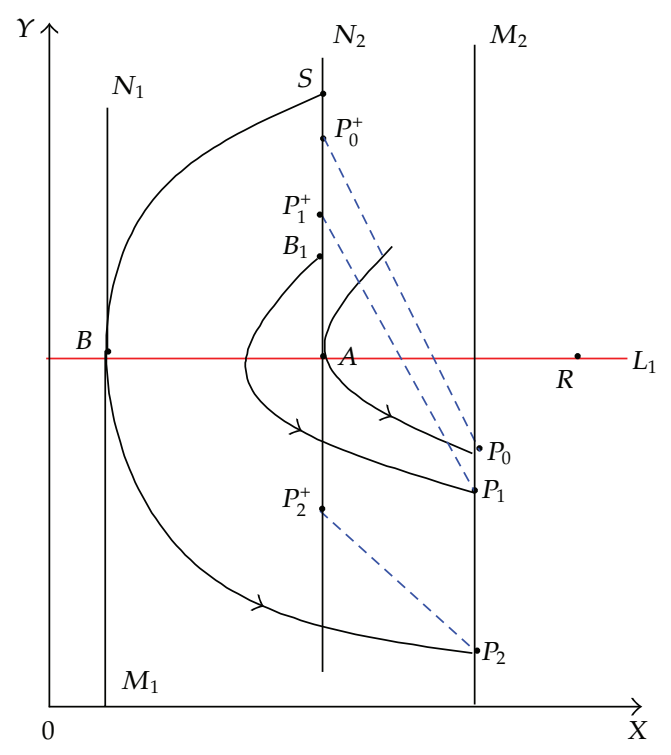

Figure 3

Subcase $2.1\left(0<h_{1}<(1-\alpha) h_{2}<h_{2}\right)$. In this case, the set $N_{2}$ is in the right side of $M_{1}$ (as shown in Figure 3). The trajectory passing through point $A$ which tangents to $N_{2}$ at point $A$ intersects with the set $M_{2}$ at point $P_{0}\left(h_{2}, y_{p_{0}}\right)$. Since the point $P_{0} \in M_{2}$, then impulse occurs at point $P_{0}$, supposing the point $P_{0}$ is subject to impulsive effects to point $P_{0}^{+}\left((1-\alpha) h_{2}, y_{P_{0}^{+}}\right)$, where $y_{P_{0}^{+}}=(1-\beta) y_{P_{0}}+q$, the position of $P_{0}^{+}$has the following three cases.

(1) $(1-\beta) y_{P_{0}}+q>r / c$ : Take a point $B_{1}\left((1-\alpha) h_{2}, \varepsilon+r / c\right) \in N_{2}$ above $A$, where $\varepsilon>0$ is small enough. Then there must exist a trajectory passing through the point $B_{1}$ which intersects with $M_{2}$ at point $P_{1}\left(h_{2}, y_{P_{1}}\right)$. In view of continuous dependence of the solution on initial value and time, we know that $y_{P_{1}}<y_{P_{0}}$, and the point $P_{1}$ is close to $P_{0}$ enough, so we have the point $P_{1}^{+}$close to $P_{0}^{+}$enough and $y_{P_{1}^{+}}<y_{P_{0}^{+}}$, then we obtain $f\left(B_{1}\right)=y_{P_{1}^{+}}-y_{B_{1}}>0$. On the other hand, the trajectory passing through point $B$ which tangents to $N_{1}$ at point $B$ intersect with $N_{2}$ at point $S$. Set $F(S)=P_{2}\left(h_{2}, y_{P_{2}}\right) \in M_{2}$. Denote the coordinates of impulsive point $P_{2}^{+}\left((1-\alpha) h_{2}, y_{P_{2}^{+}}\right)$corresponding to the point $P_{2}\left(h_{2}, y_{P_{2}}\right)$. If $y_{S} \geq y_{P_{0}^{+}}$, then $y_{P_{2}^{+}}<y_{P_{0}^{+}}$. So we obtain $f(S)=y_{P_{2}^{+}}-y_{S}<0$. There exists an order one periodic solution of system (1.3), whose initial point is between the point $B_{1}$ and the point $S$ in set $N_{2}$. If $y_{S}<y_{P_{0}^{+}}$and $y_{P_{2}^{+}}>y_{S}$, from the vector field of system (1.3), we know that the trajectory of system (1.3) with any initiating point on the $N_{2}$ will ultimately stay in $\Gamma_{1}$ after one impulsive effect (as shown in Figure 4). Therefore, there is no an order one periodic solution of system (1.3);

(2) $(1-\beta) y_{P_{0}}+q<r / c$ (as shown in Figure 5): In this case, the point $P_{0}^{+}$lies below the point $A$, that is, $(1-\beta) y_{P_{0}}+q<r / c$, thus the successor function of the point $A$ is $f(A)=(1-\beta) y_{P_{0}}+q-r / c<0$. Take another point $B_{1}\left((1-\alpha) h_{2}, \varepsilon\right) \in N_{2}$, where $\varepsilon>0$ is small enough. Then there must exist a trajectory passing through the point $B_{1}$ which intersects with $M_{2}$ at a point $P_{1}\left(h_{2}, y_{P_{1}}\right) \in M_{2}$. Supposing the point 


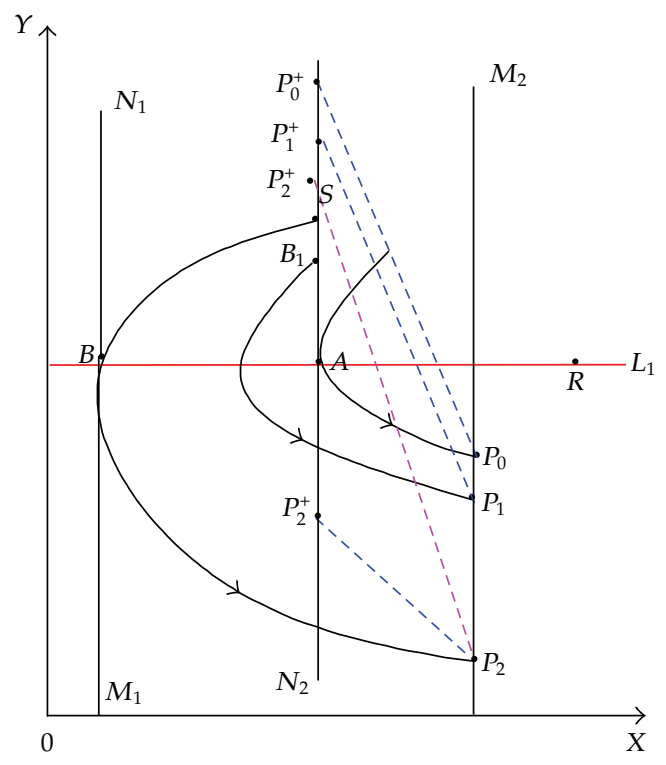

Figure 4

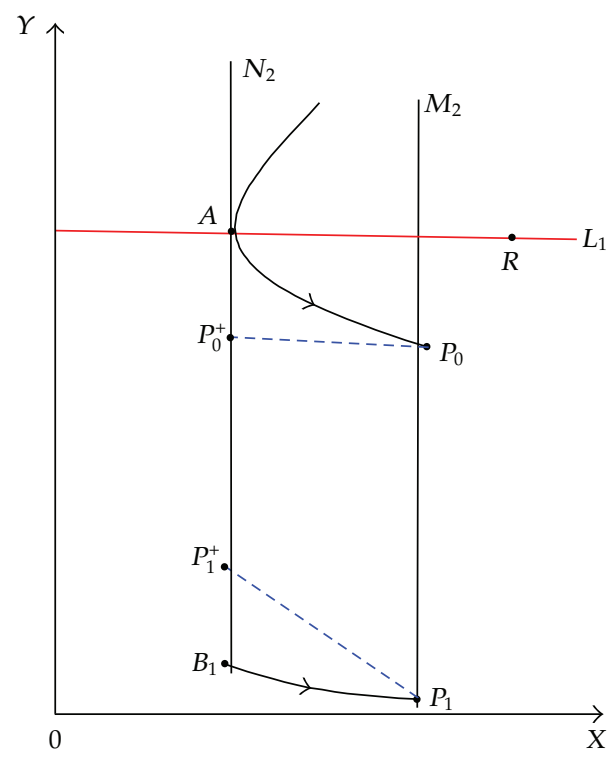

Figure 5

$P_{1}\left(h_{2}, y_{P_{1}}\right)$ is subject to impulsive effects to point $P_{1}^{+}\left((1-\alpha) h_{2}, y_{P_{1}^{+}}\right)$, then we have $y_{P_{1}^{+}}>\varepsilon$, so we have $f\left(C_{1}\right)=y_{P_{1}^{+}}-\varepsilon>0$. From Lemma 2.3, there exists an order one periodic solution of system(1.3), whose initial point is between $B_{1}$ and $A$ in set $N_{2}$;

(3) $(1-\beta) y_{P_{0}}+q=r / c: P_{0}^{+}$coincides with $A$, and the successor function of $A$ is that $f(A)=0$, so there exists an order one periodic solution of system (1.3) which is just a part of the trajectory passing through the $A$. 


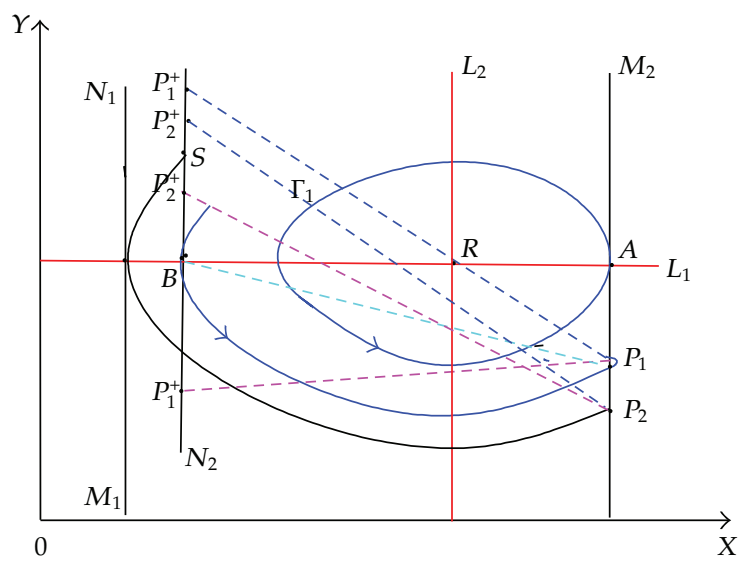

Figure 6

Now we can summarize the above results in the following theorem.

Theorem 3.3. Assuming that $d<e c x_{0}, 0<h_{1}<(1-\alpha) h_{2}<h_{2}<d / e c$.

If $(1-\beta) y_{P_{0}}+q \leq r / c$, there exists an order one periodic solutions of the system (1.3).

If $(1-\beta) y_{P_{0}}+q>r / c$ and $y_{S} \geq y_{P_{0}^{+}}$or $y_{S}<y_{P_{0}^{+}}$and $y_{P_{2}^{+}} \leq y_{S}$, there exists an order one periodic solutions of the system (1.3).

Subcase $2.2\left(0<(1-\alpha) h_{2}<h_{1}<h_{2}\right)$. In this case, the set $N_{2}$ is in the left side of $N_{1}$. Any trajectory from initial point $\left(x_{0}^{+}, y_{0}^{+}\right) \in N_{2}$ will intersect with $M_{1}$ at some point with time increasing. Like the analysis of Case 1 , the trajectory from initial point $\left(x_{0}^{+}, y_{0}^{+}\right) \in N_{2}$ on the set $N_{2}$ will stay in the region $\Omega_{1}=\left\{(x, y) \mid x \geq 0, y \geq 0, x \leq h_{1}\right\}$. Similarly, any trajectory from initial point $\left(x_{0}^{+}, y_{0}^{+}\right) \in \Omega_{0}=\left\{(x, y) \mid x \geq 0, y \geq 0, x \leq h_{2}\right\}$ will stay in the region $\Omega_{1}$ after one impulsive effect or free from impulsive effect.

Theorem 3.4. If $d<e c x_{0}$ and $0<(1-\alpha) h_{2}<h_{1}<h_{2}<d / e c$, there is no order one periodic solutions to the system (1.3), and the trajectory with initial point $\left(x_{0}^{+}, y_{0}^{+}\right) \in \Omega_{0}=\{(x, y) \mid x \geq$ $\left.0, y \geq 0, x \leq h_{2}\right\}$ will stay in the region $\Omega_{1}=\left\{(x, y) \mid x \geq 0, y \geq 0, x \leq h_{1}\right\}$.

Case $3\left(d / e c<h_{2} \leq x_{0}\right)$. In this case, the set $M_{2}$ is in the right side of stable center $R$. In the light of the different position of $N_{2}$, we consider the following two subject cases.

Subcase $3.1\left(h_{1}<(1-\alpha) h_{2}\right)$. In this case, the set $M_{2}$ is in the right side of $R$. Then there exists a unique closed trajectory $\Gamma_{1}$ of system (1.3) which contains the point $R$ and is tangent to $M_{2}$ at the point $A$.

Since $\Gamma_{1}$ is a closed trajectory, we take the minimal value $\delta_{\min }$ of abscissas at the trajectory $\Gamma_{1}$, namely, $\delta_{\min } \leq x$ holds for any abscissas of $\Gamma_{1}$.

(1) $h_{1}<(1-\alpha) h_{2}<\delta_{\min }$ : In this case, there is a trajectory, which contains the point $R$ and is tangent to the $N_{2}$ at the point $B$ intersecting with $M_{2}$ at a point $P_{1}\left(h_{2}, y_{P_{1}}\right) \in M_{2}$. Suppose point $P_{1}$ is subject to impulsive effects to point $P_{1}^{+}\left((1-\alpha) h_{2}, y_{P_{1}^{+}}\right)$, here $y_{P_{1}^{+}}=(1-\beta) y_{P_{1}}+q$. Like the analysis of Subcase 2.1 , we can prove that there exists an order one periodic solution to the system (1.3) in this case (as shown in Figure 6); 


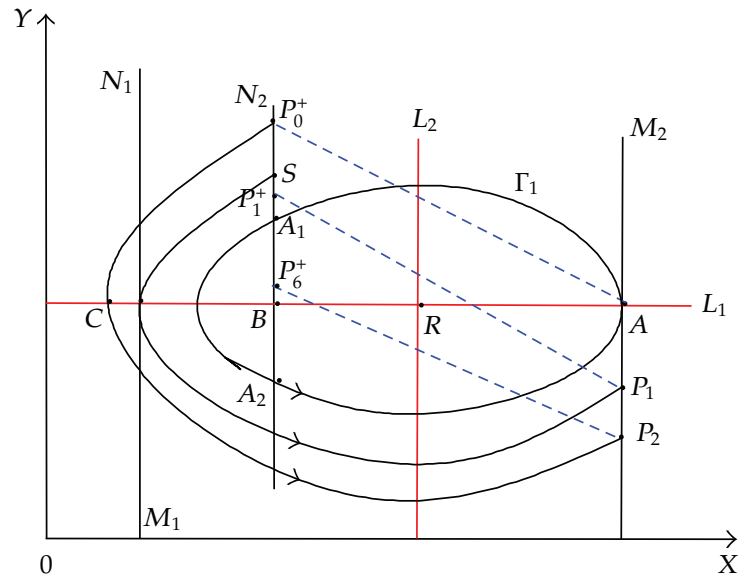

Figure 7

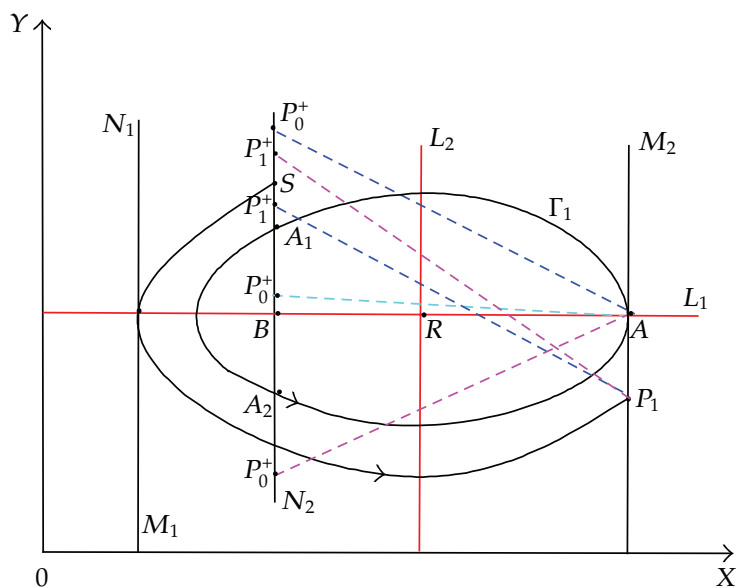

Figure 8

(2) $h_{1}<\delta_{\min }<(1-\alpha) h_{2}$ : In this case, denote the closed trajectory $\Gamma_{1}$ of system (1.3) intersecting with the set $N_{2}$ two point $A_{1}\left((1-\alpha) h_{2}, y_{A_{1}}\right)$ and $A_{2}\left((1-\alpha) h_{2}, y_{A_{2}}\right)$ (as shown in Figure 7). Since $A \in M_{2}$, impulse occurs at the point $A$. Suppose point $A$ is subject to impulsive effects to point $P_{0}^{+}\left((1-\alpha) h_{2}, y_{P_{0}^{+}}\right)$, here $y_{P_{0}^{+}}=(1-\beta)(r / c)+q$. If $(1-\beta)(r / c)+q<y_{A_{2}}$, the point $P_{0}^{+}$lies below the point $A_{2}$. Like the analysis of (2) of Subcase 2.1, we can prove that there exists an order one periodic solution to the system (1.3) in this case. If $(1-\beta)(r / c)+q>y_{A_{1}}$, the point $P_{0}^{+}$is above the point $A_{1}$. Suppose the trajectory passing through point $B$ which tangents to $N_{1}$ at point $B$ intersects with $N_{2}$ at a point $S$. Like the analysis of (1) of Subcase 2.1, we obtain sufficient conditions of existence of order one periodic solution to the system (1.3);

(3) $y_{A_{2}}<(1-\beta)(r / c)+q<y_{A_{1}}$ : In this case, we note that the point $P_{0}^{+}$must lie between the point $A_{1}$ and the point $A_{2}$ (as shown in Figure 8). Take a point $B_{1} \in M_{2}$ such that $B_{1}$ jumps to $A_{2}$ after the impulsive effect and denote $A_{2}=B_{1}^{+}$. Since $y_{P_{0}^{+}}>y_{B_{1}^{+}}$, we have $y_{A}>y_{B_{1}}$. Let $R\left(B_{1}\right)=B_{2}^{+} \in N_{2}$, take a point $B_{2} \in M_{2}$ such that $B_{2}$ jumps 


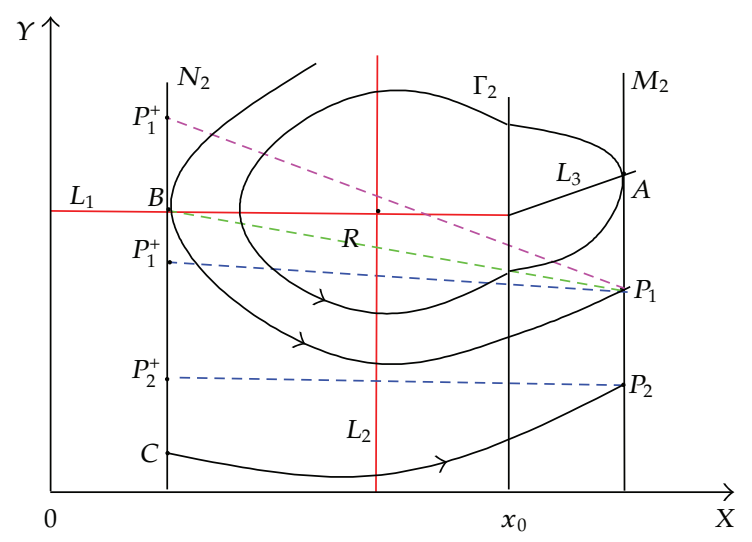

Figure 9

to $B_{2}^{+}$after the impulsive effects, then we have $y_{B_{1}^{+}}>y_{B_{2}^{+}}, y_{B_{1}}>y_{B_{2}}$. This process continues until there exists a $B_{K}^{+} \in N_{2}\left(K \in Z_{+}\right)$satisfying $y_{B_{k}^{+}}<q$. So we obtain a sequence $\left\{B_{k}^{+}\right\}_{k=1,2, \ldots, K}$ of set $M_{2}$ and a sequence $\left\{B_{k}\right\}_{k=1,2, \ldots, K}$ of set $N_{2}$ satisfying $R\left(B_{k-1}\right)=B_{k}^{+} \in N_{2}, y_{B_{k-1}^{+}}>y_{B_{k}^{+}}$. In the following, we will prove that the trajectory of system (1.3) with any initiating point of set $N_{2}$ will ultimately stay in $\Gamma_{1}$. From the vector field of system (1.3), we know the trajectory of system (1.3) with any initiating point between the point $A_{1}$ and $A_{2}$ will be free from impulsive effect and ultimately will stay in $\Gamma_{1}$. For any point below $A_{2}$, it must lie between $B_{k}^{+}$and $B_{k-1}^{+}$, here $k=2,3 \ldots, K+1$ and $A_{2}=B_{1}^{+}$. After $k$ times' impulsive effects, the trajectory with this initiating point will arrive at some point of the set $N_{2}$ which must be between $A_{1}$ and $A_{2}$, and then ultimately stay in $\Gamma_{1}$. Denote the intersection of the trajectory passing through the point $B$ which tangents to $N_{1}$ at point $B$ with the set $N_{2}$ at point $S\left((1-\alpha) h_{2}, y_{S}\right)$ (Figure 7). The trajectory of system (1.3) with any initiating point on segment $\overline{A_{1} S}$ intersects with the set $N_{2}$ at some point below $A_{2}$ with time increasing, so just like the analysis above we obtain it will ultimately stay in $\Gamma_{1}$. Therefore, for any point below $S$ will ultimately stay in region $\Gamma_{1}$ with time increasing.

Now we can summarize the above results as the following theorem.

Theorem 3.5. Assuming that $d<e c x_{0}, h_{1}<\delta_{\min }<(1-\alpha) h_{2}<d / e c<h_{2} \leq x_{0}$ and $y_{A_{2}}<$ $(1-\beta)(r / c)+q<y_{A_{1}}$, there is no periodic solution in system (1.3) and the trajectory with any initiating point below $S$ will stay in $\Gamma_{1}$ or in the region $\Omega_{1}=\left\{(x, y) \mid x \geq 0, y \geq 0, x \leq h_{1}\right\}$.

Case $4\left(x_{0}<h_{2}\right)$. In this case, denote the intersection of the line $L_{1}$ with the set $N$ at point $B\left((1-\alpha) h_{2}, r / c\right)$, and the intersection between the line $L_{3}$ and the set $M_{2}$ at point $A\left(h_{2}, r h_{2} / c x_{0}\right)$ (as shown in Figure 9). By Lemma 2.3 and means of qualitative analysis, there exists a unique closed trajectory $\Gamma_{2}$ of system (1.3) which is tangent to the set $M_{2}$ at the point $A$ and has minimal value $\lambda_{\min }$ at the line $L_{1}$. In the light of the different position of the set $N_{2}$, we consider the following two cases.

Subcase $4.1\left(0<h_{1}<(1-\alpha) h_{2}<\lambda_{\min }\right)$. In this case, there exists a unique trajectory of system (1.3) which is tangent to the set $N_{2}$ at the point $B$. Set $F(B)=P_{1} \in M_{2}$, then pulse occurs 
at point $P_{1}$, the impulsive function transfers the point $P_{1}$ into $P_{1}^{+}$. Like the analysis of (1) of Subcase 2.1, we can prove that there exists an order one periodic solution in system (1.3) in this case.

Subcase $4.2\left(h_{1}<\lambda_{\min }<(1-\alpha) h<x_{0}<h_{2}\right)$. In this case, let the closed trajectory $\Gamma_{2}$ of system (1.3) intersects $N_{2}$ at two point $A_{1}\left((1-\alpha) h, y_{A_{1}}\right)$ and $A_{2}\left((1-\alpha) h, y_{A_{2}}\right)$. Like the analysis of (2) of Subcase 3.1, we can prove that there exists an order one periodic solution in system (1.3) in this case; like the analysis of (3) of Subcase 3.1 we can prove that there is no periodic solution in system (1.3).

\section{Attractiveness of the Order One Periodic Solution}

In this section, under the condition of existence of order one periodic solution to system (1.3) and the initial value of pest population $x(0) \leq h_{2}$, we discuss its attractiveness. We focus on Cases 1 and 2; by similar method we can obtain similar results about Cases 3 and 4 .

Theorem 4.1. Assuming that $d<$ cex $x_{0}, h_{1}<h_{2}<r / c$ and $\delta \geq r / c$. If $y_{P_{0}^{+}}>y_{P_{2}^{+}}>y_{P^{+}}$or $y_{P_{0}^{+}}<y_{P_{2}^{+}}<y_{P^{+}}$, then

(I) there exists a unique order one periodic solution of system (1.3);

(II) If $(1-\alpha) h_{2}<h_{1}$, order one periodic solution of system (1.3) is attractive in the region $\Omega_{0}=\left\{(x, y) \mid x \geq 0, y \geq 0, x \leq h_{2}\right\}$.

Proof. By the derivation of Theorem 3.1, we know that there exists an order one periodic solution of system (1.3). We assume that trajectory $\widehat{P P^{+}}$and segment $\overline{P P^{+}}$formulate an order one periodic solution of system (1.3), that is, there exists a $P^{+} \in N_{2}$ such that the successor function of $P^{+}$satisfies $f\left(P^{+}\right)=0$. First, we will prove the uniqueness of the order one periodic solution.

We take any two points $C_{1}\left(h_{1}, y_{C_{1}}\right) \in N_{1}, C_{2}\left(h_{1}, y_{C_{2}}\right) \in N_{1}$ satisfying $y_{C_{2}}>y_{C_{1}}>y_{A}$, then we obtain two trajectories whose initiate points are $C_{1}$ and $C_{2}$ intersect with the set $M_{1}$ at two points $D_{1}\left(h_{1}, y_{D_{1}}\right)$ and $D_{2}\left(h_{1}, y_{D_{2}}\right)$, respectively (Figure 10$)$. In view of the vector field of system (1.3) and the disjointness of any two trajectories without impulse, we know that $y_{D_{1}}>y_{D_{2}}$. Suppose the points $D_{1}$ and $D_{2}$ are subject to impulsive effect to points $D_{1}^{+}\left(h_{1}, y_{D_{1}^{+}}\right)$ and $D_{2}^{+}\left(h_{2}, y_{D_{2}^{+}}\right)$, respectively, then we have $y_{D_{1}^{+}}>y_{D_{2}^{+}}$and $f\left(C_{1}\right)=y_{D_{1}^{+}}-y_{C_{1}}, f\left(C_{2}\right)=y_{D_{2}^{+}}-y_{C_{2}}$, so we get $f\left(C_{1}\right)-f\left(C_{2}\right)<0$, thus we obtain that the successor function $f(x)$ is decreasing monotonously in $N_{1}$; therefore there is a unique point $P^{+} \in N_{1}$ satisfying $f\left(P^{+}\right)=0$, and the trajectory $\widehat{P^{+} P P^{+}}$is a unique order one periodic solution of system (1.3).

Next we prove the attractiveness of the order one periodic solution $\widehat{P^{+} P P^{+}}$in the region $\Omega_{0}=\left\{(x, y) \mid x \geq 0, y \geq 0, x \leq h_{2}\right\}$. We focus on the case $y_{P_{0}^{+}}>y_{P_{2}^{+}}>y_{P^{+}} ;$by similar method we can obtain similar results about case $y_{P_{0}^{+}}<y_{P_{2}^{+}}<y_{P^{+}}$(Figure 11).

Take any point $P_{0}^{+}\left(h_{1}, y_{P_{0}^{+}}\right) \in N_{1}$ above $P^{+}$. Denote the first intersection point of the trajectory from initiating point $P_{0}^{+}\left(h_{1}, y_{P_{0}^{+}}\right)$with the set $M_{1}$ at $P_{1}\left(h_{1}, y_{P_{1}}\right)$, and the corresponding consecutive points are $P_{2}\left(h_{1}, y_{P_{2}}\right), P_{3}\left(h_{1}, y_{P_{3}}\right), P_{4}\left(h_{1}, y_{P_{4}}\right), \ldots$, respectively. Consequently, under the effect of impulsive function $I$, the corresponding points after pulse are $P_{1}^{+}\left(h_{1}, y_{P_{1}^{+}}\right), P_{2}^{+}\left(h_{1}, y_{P_{2}^{+}}\right), P_{3}^{+}\left(h_{1}, y_{P_{3}^{+}}\right), \ldots$ 


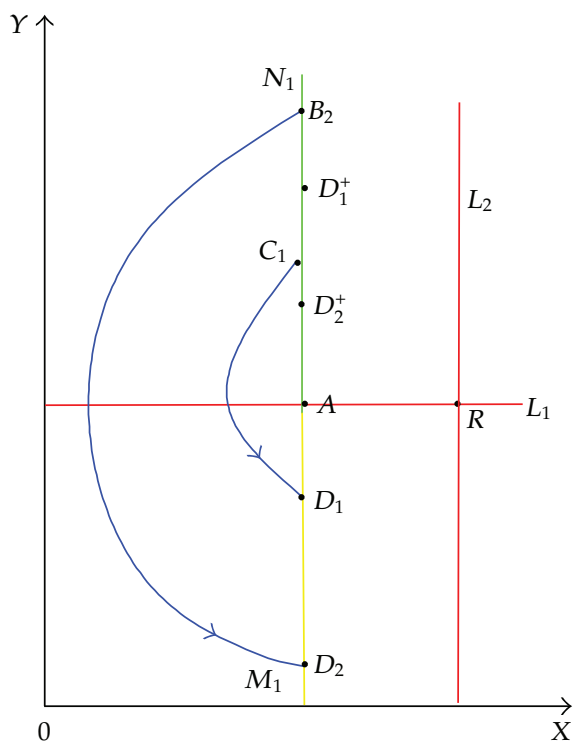

Figure 10

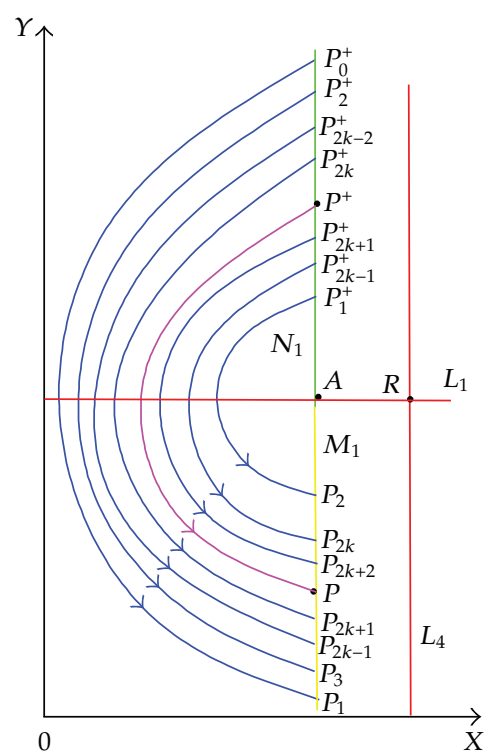

Figure 11

Due to conditions $y_{P_{0}^{+}}>y_{P_{2}^{+}}>y_{P^{+}}, y_{P_{k}^{+}}=y_{P_{k}}+\delta, \delta \geq a / b$ and disjointness of any two trajectories, we get a sequence $\left\{P_{k}^{+}\right\}_{k=1,2, \ldots}$ of the set $N_{1}$ satisfying

$$
y_{P_{1}^{+}}<y_{P_{3}^{+}}<\cdots<y_{P_{2 k-1}^{+}}<y_{P_{2 k+1}^{+}}<\cdots<y_{P^{+}}<\cdots<y_{P_{2 k}^{+}}<y_{P_{2 k-2}^{+}}<\cdots<y_{P_{2}^{+}}<y_{P_{0}^{+}}
$$




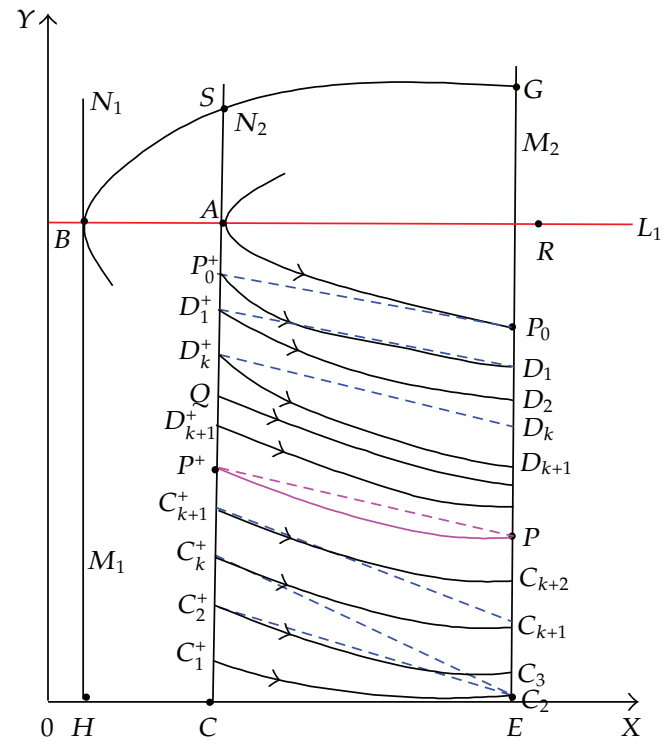

Figure 12

So the successor function $f\left(P_{2 k-1}^{+}\right)=y_{P_{2 k}^{+}}-y_{P_{2 k-1}^{+}}>0$ and $f\left(P_{2 k}^{+}\right)=y_{P_{2 k+1}^{+}}-y_{P_{2 k}^{+}}<0$ hold. Series $\left\{y_{P_{2 k-1}}\right\}_{k=1,2, \ldots}$ increases monotonously and has upper bound, so $\lim _{k \rightarrow \infty} y_{P_{2 k-1}^{+}}$exists. Next we will prove $\lim _{k \rightarrow \infty} y_{P_{2 k-1}^{+}}=y_{P^{+}}$. Set $\lim _{k \rightarrow \infty} P_{2 k-1}=C^{+}$, we will prove $P^{+}=C^{+}$. Otherwise $P^{+} \neq C^{+}$, then there is a trajectory passing through the point $C^{+}$which intersects the set $M_{1}$ at point $\widetilde{C}$, then we have $y_{\tilde{C}}>y_{P}, y_{\tilde{C}^{+}}>y_{P^{+}}$. Since $f\left(C^{+}\right) \geq 0$ and $P^{+} \neq C^{+}$, according to the uniqueness of the periodic solution, then we have $f\left(C^{+}\right)=y_{\tilde{C}^{+}}-y_{C^{+}}>0$, thus $y_{C^{+}}<y_{P^{+}}<y_{\tilde{C}^{+}}$ hold. Analogously, let trajectory passing through the point $C^{+}$which intersects the set $M_{1}$ at

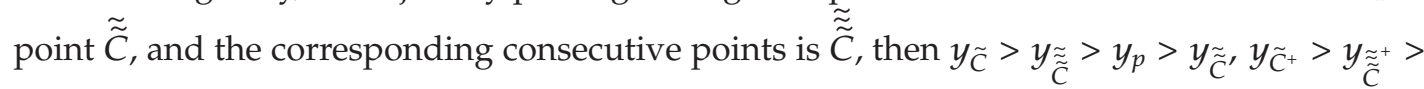
$y_{\tilde{\tilde{C}}^{+}}>y_{p^{+}}>y_{C^{+}}$, then we have $f\left(\widetilde{\widetilde{C}}^{+}\right)=y_{\tilde{\tilde{C}}^{+}}-y_{\tilde{C}^{+}}>0$, this contradicts to the fact that $C^{+}$is a limit of sequence $\left\{P_{2 k-1}^{+}\right\}_{k=1,2, \ldots .}$, so we obtain $P^{+}=C^{+}$. Therefore, we have $\lim _{k \rightarrow \infty} y_{P_{2 k-1}^{+}}=y_{P^{+}}$. Similarly, we can prove $\lim _{k \rightarrow \infty} y_{P_{2 k}^{+}}=y_{P^{+}}$.

From above analysis, we know that there exists a unique order one periodic solution in system (1.3), and the trajectory from initiating any point of the $N_{1}$ will ultimately tend to be order one periodic solution $\widehat{P^{+} P P^{+}}$.

Any trajectory from initial point $\left(x_{0}^{+}, y_{0}^{+}\right) \in \Omega_{0}=\left\{(x, y) \mid x \geq 0, y \geq 0, x \leq h_{2}\right\}$ will intersect with $N_{1}$ at some point with time increasing on the condition that $(1-\alpha) h_{2}<h_{1}<$ $h_{2}<d / b(\lambda-d h)$; therefore, the trajectory from initial point on $N_{1}$ ultimately tends to be order one periodic solution $\widehat{P^{+} P P^{+}}$. Therefore, order one periodic solution $\widehat{P^{+} P P^{+}}$is attractive in the region $\Omega_{0}$. This completes the proof.

Remark 4.2. Assuming that $d<e c x_{0}, h_{1}<h_{2}<d / e c$ and $\delta \geq r / c$, if $y_{P^{+}}<y_{P_{0}^{+}}<y_{P_{2}^{+}}$or $y_{P^{+}}>y_{P_{0}^{+}}>y_{P_{2}^{+}}$, then the order one periodic solution is unattractive.

Theorem 4.3. Assuming that $d<e c x_{0}, h_{1}<(1-\alpha) h_{2}<h_{2}<d / e c$ and $y_{P_{0}^{+}}<y_{A}$ (as shown in Figure 12), then 
(I) there exists an odd number of order one periodic solutions of system (1.3) with initial value between $\mathrm{C}_{1}^{+}$and $A$ in set $\mathrm{N}_{2}$;

(II) if $(1-\alpha) h_{2}<h_{1}$ and the periodic solution is unique, then the periodic solution is attractive in region $\Omega_{2}$, here $\Omega_{2}$ is open region which is constituted by trajectory $\widehat{G B}$, segment $\overline{B H}$, segment $\overline{H E}$, and segment $\overline{E G}$.

Proof. (I) due to the (2) of Subcase 2.1, $f(A)<0$ and $f\left(C_{1}^{+}\right)>0$ and the continuous successor function $f(x)$, there exists an odd number of root satisfying $f(x)=0$, then we can get that there exists an odd number of order one periodic solutions of system (1.3) with initial value between $C_{1}^{+}$and $A$ in set $N_{2}$;

(II) by the derivation of Theorem 3.3, we know that there exists an order one periodic solution of system (1.3) whose initial point is between $C_{1}^{+}$and $P_{0}^{+}$in the set $N_{2}$. Assume trajectory $\widehat{P^{+} P}$ and segment $\overline{P P^{+}}$formulate the unique order one periodic solution of system (1.3) with initial point $P^{+} \in N_{2}$.

On the one hand, take a point $C_{1}^{+}\left((1-\alpha) h_{2}, y_{C_{1}^{+}}\right) \in N_{2}$ satisfying $y_{C_{1}^{+}}=\varepsilon<q$ and $y_{C_{1}^{+}}<y_{P^{+}}$. The trajectory passing through the point $C_{1}^{+}\left((1-\alpha) h_{2}, \varepsilon\right)$ which intersects with set $M_{2}$ at point $C_{2}\left(h_{2}, y_{C_{2}}\right)$, that is, $F\left(C_{1}^{+}\right)=C_{2} \in M_{2}$, then we have $y_{C_{2}}<y_{P}$, thus $y_{C_{2}^{+}}<y_{P^{+}}$. Since $y_{C_{2}^{+}}=(1-\beta) y_{C_{2}}+q>\varepsilon$, so we obtain $f\left(C_{1}^{+}\right)=y_{C_{2}^{+}}-y_{C_{1}^{+}}=y_{C_{2}^{+}}-\varepsilon>0$; set $F\left(C_{2}^{+}\right)=C_{3} \in M_{2}$, because $y_{C_{1}^{+}}<y_{C_{2}^{+}}<y_{P^{+}}$, we know that $y_{C_{2}}<y_{C_{3}}<y_{P}$, then we have $y_{C_{2}^{+}}<y_{C_{3}^{+}}<y_{P^{+}}$and $f\left(C_{2}^{+}\right)=y_{C_{3}^{+}}-y_{C_{2}^{+}}>0$. This process is continuing, then we get a sequence $\left\{C_{k}^{+}\right\}_{k=1,2, \ldots}$ of the set $N_{2}$ satisfying

$$
y_{C_{1}^{+}}<y_{C_{2}^{+}}<\cdots<y_{C_{k}^{+}}<\cdots<y_{P^{+}}
$$

and $f\left(C_{k}^{+}\right)=y_{C_{k+1}^{+}}-y_{C_{k}^{+}}>0$. Series $\left\{y_{C_{k}^{+}}\right\}_{k=1,2, \ldots}$ increases monotonously and has upper bound, so $\lim _{k \rightarrow \infty} y_{C_{k}^{+}}$exists. Like the proof of Theorem 4.1, we can prove $\lim _{k \rightarrow \infty} y_{C_{k}^{+}}=y_{P^{+}}$.

On the other hand, set $F\left(P_{0}^{+}\right)=D_{1} \in M_{2}$, then $D_{1}$ jumps to $D_{1}^{+} \in N_{2}$ under the impulsive effects. Since $y_{P^{+}}<y_{P_{0}^{+}}<y_{A}$, we have $y_{P}<y_{D_{1}}<y_{P_{0}}$, thus we obtain $y_{P^{+}}<$ $y_{D_{1}^{+}}<y_{P_{0}^{+}}, f\left(P_{0}^{+}\right)=y_{D_{1}^{+}}-y_{P_{0}^{+}}<0$. Set $F\left(D_{1}^{+}\right)=D_{2} \in M_{2}$, then $D_{2}$ jumps to $D_{2}^{+} \in N_{2}$ under the impulsive effects. We have $y_{P^{+}}<y_{D_{2}^{+}}<y_{D_{1}^{+}}$. This process is continuing, we can obtain a sequence $\left\{D_{k}^{+}\right\}_{k=1,2 \ldots}$ of the set $N_{2}$ satisfying

$$
y_{P_{0}^{+}}>y_{D_{1}^{+}}>y_{D_{2}^{+}}>\cdots>y_{D_{k}^{+}}>\cdots>y_{P^{+}}
$$

and $f\left(D_{k}^{+}\right)=y_{D_{k+1}^{+}}-y_{D_{k}^{+}}<0$. Series $\left\{y_{D_{k}^{+}}\right\}_{k=1,2, \ldots}$ decreases monotonously and has lower bound, so $\lim _{k \rightarrow \infty} y_{D_{k}^{+}}$exists. Similarly, we can prove $\lim _{k \rightarrow \infty} y_{D_{k}^{+}}=y_{P^{+}}$.

Any point $Q \in N_{2}$ below $A$ must be in some interval $\left[y_{D_{k+1}^{+}}, y_{D_{k}^{+}}\right)_{k=1,2, \ldots,},\left[y_{D_{1}^{+}}, y_{P_{0}^{+}}\right)$, $\left[y_{P_{0}^{+}}, y_{A}\right),\left[y_{C_{k}^{+}}, y_{C_{k+1}^{+}}\right)_{k=1,2, \ldots .}$. Without loss of generality, we assume that the point $Q \in$ $\left[y_{D_{k+1}^{+}}, y_{D_{k}^{+}}\right)$. The trajectory with initiating point $Q$ moves between trajectory $\widehat{D_{k}^{+} D_{k+1}}$ and $D_{k+1}^{+} D_{k+2}$ and intersects with $M_{2}$ at some point between $D_{k+2}$ and $D_{k+1}$, under the impulsive effects it jumps to the point of $N_{2}$ which is between $\left[y_{D_{k+2}^{+}}, y_{D_{k+1}^{+}}\right)$, then trajectory $\widetilde{\Pi}(Q, t)$ continues to move between trajectory $\widehat{D_{k+1}^{+} D_{k+2}}$ and $\widehat{D_{k+2}^{+} D_{k+3}}$. This process can be continued 
unlimitedly. Since $\lim _{k \rightarrow \infty} y_{D_{k}^{+}}=y_{P^{+}}$, the intersection sequence of trajectory $\widetilde{\Pi}(Q, t)$ with the set $N_{2}$ will ultimately tend to be the point $P^{+}$. Similarly, if $Q \in\left[y_{C_{k}^{+}}, y_{C_{k+1}^{+}}\right)$, we also can get the intersection sequence of trajectory $\widetilde{\Pi}(Q, t)$, and the set $N_{2}$ will ultimately tend to be point $P^{+}$. Thus the trajectory from initiating any point below $A$ ultimately tends to be the unique order one periodic solution $\widehat{P^{+} P P^{+}}$.

Denote the intersection of the trajectory passing through the point $B$ which tangents to $N_{1}$ at the point $B$, and the set $N_{2}$ by a point $S\left((1-\alpha) h_{2}, y_{S}\right)$. The trajectory from any initiating point on segment $\overline{A S}$ will intersect with the set $N_{2}$ at some point below $A$ with time increasing, so like the analysis above we obtain that the trajectory from any initiating point on segment $\overline{A S}$ will ultimately tend to be the unique order one periodic solution $\widehat{P^{+} P P^{+}}$.

Since the trajectory with any initiating point of the $\Omega_{2}$ will definitely intersect with set $N_{2}$. From the above analysis, we know that the trajectory with any initiating point on segment $\overline{A S}$ will ultimately tend to be order one periodic solution $\widehat{P^{+} \widehat{P P}^{+}}$. Therefore, the unique order one periodic solution $\widehat{P^{+} P P^{+}}$is attractive in the region $\Omega_{2}$. This completes the proof.

Remark 4.4. Assuming that $d<e c x_{0}, h_{1}<(1-\alpha) h_{2}<h_{2}<d / e c$ and $y_{C_{1}^{+}}<y_{A}<y_{P_{0}^{+}}$, the order one periodic solution with initial point between $A$ and $P_{0}^{+}$is unattractive.

\section{Conclusion}

In this paper, a state-dependent impulsive dynamical model with Holling I functional response predator-prey concerning different control methods at different thresholds is proposed; we find a new method to study existence and attractiveness of order one periodic solution of such system. We define semicontinuous dynamical system and successor function and demonstrate the sufficient conditions that system (1.3) exists order one periodic solution with differential geometry theory and successor function. Besides, we successfully prove the attractiveness of the order one periodic solution by sequence convergence rules and qualitative analysis. In order to testify the validity of our results, we consider the following example:

$$
\begin{gathered}
x^{\prime}(t)=0.4 x(t)-0.6 x(t) y(t), \quad x \leq 0.8, \\
y^{\prime}(t)=-0.2 y(t)+0.3 x(t) y(t), \quad \\
x^{\prime}(t)=0.4 x(t)-0.48 y(t), \quad x>0.8, \\
y^{\prime}(t)=-0.6 y(t)+0.24 y(t), \quad \\
\Delta x(t)=0, \quad x=h_{1}, h_{2} \text { or } x=h_{1}, y>y^{*}, \\
\Delta y(t)=0.8, \quad y y^{*}, \\
\Delta x(t)=-0.5 x(t), \quad x=h_{2}, \\
\Delta y(t)=-0.2 y(t)+0.5, \quad
\end{gathered}
$$

where $0<h_{1}<h_{2}<x^{*}$. Now, we consider the impulsive effects influences on the dynamics of system (5.1).

Example 5.1. Existence and attractiveness of order one periodic solution. 


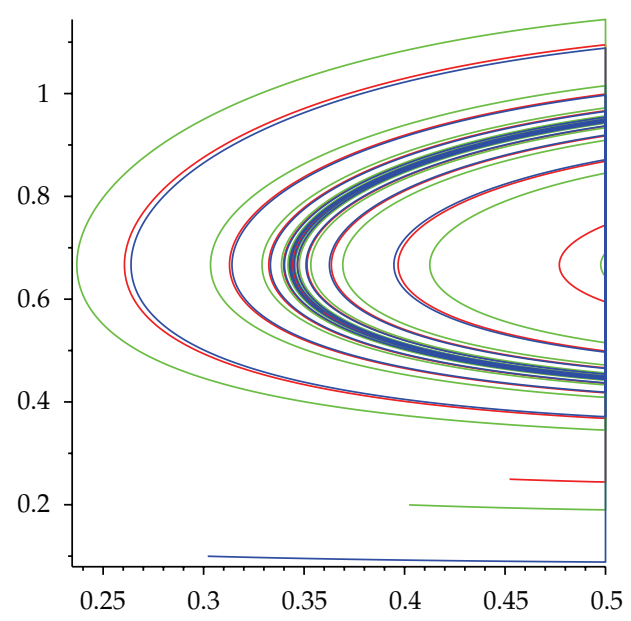

(a)

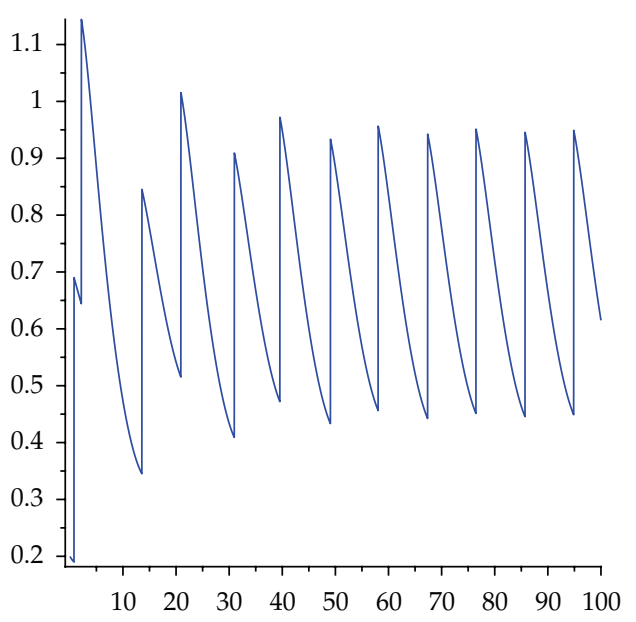

(b)

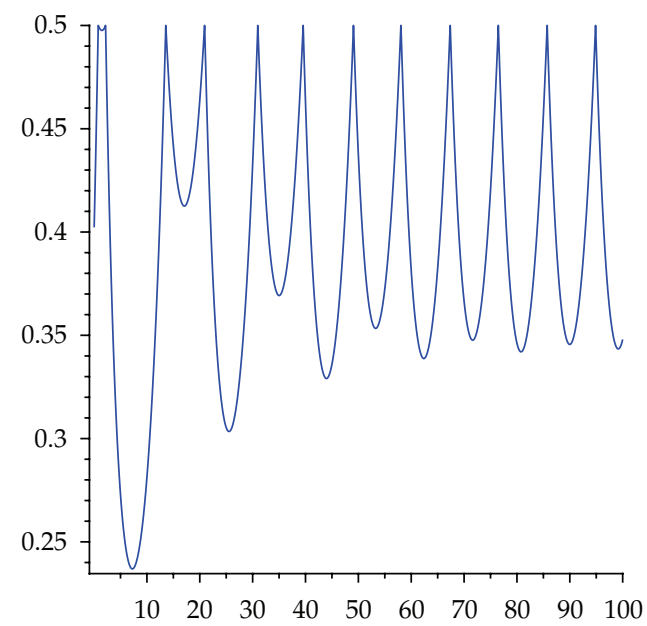

(c)

Figure 13: The time series and phase diagram for system (1.3) starting from initial value $(0.45,0.25)$ (red), $(0.4,0.2)$ (green), and $(0.3,0.1)$ (blue) $0<h_{1}<h_{2}<x^{*}$.

We set $h_{2}=0.6,(1-\alpha) h_{2}<h_{2}<x^{*}$, initiating points are $(0.45,0.25)($ red $),(0.4,0.2)$ (green), and $(0.3,0.1)$ (blue), respectively. Figure 13 shows that the conditions of Theorems 3.1 and 4.1 hold, system (1.3) exists order one periodic solution, and the trajectory from different initiating must ultimately tend to be the order one periodic solution. Therefore, order one periodic solution is attractive.

Example 5.2. Existence and attractiveness of positive periodic solution.

We set $h_{1}=0.3, h_{2}=0.6, h_{1}<(1-\alpha) h_{2}<x^{*}<h_{2}<x_{0}$, initiating points are $(0.45,0.25)$ (red), $(0.4,0.2)$ (green), and $(0.3,0.1)$ (blue), respectively. Figure 14 shows that the conditions of Theorems 3.3 and 4.3 hold, system (1.3) exists order one periodic solution, 


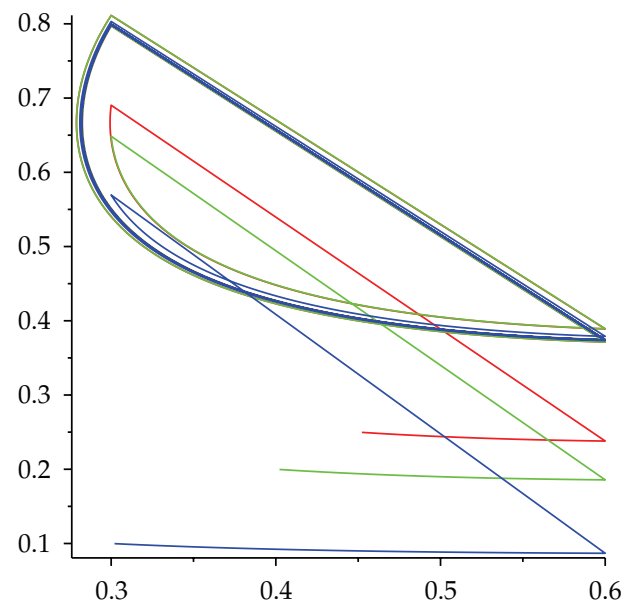

(a)

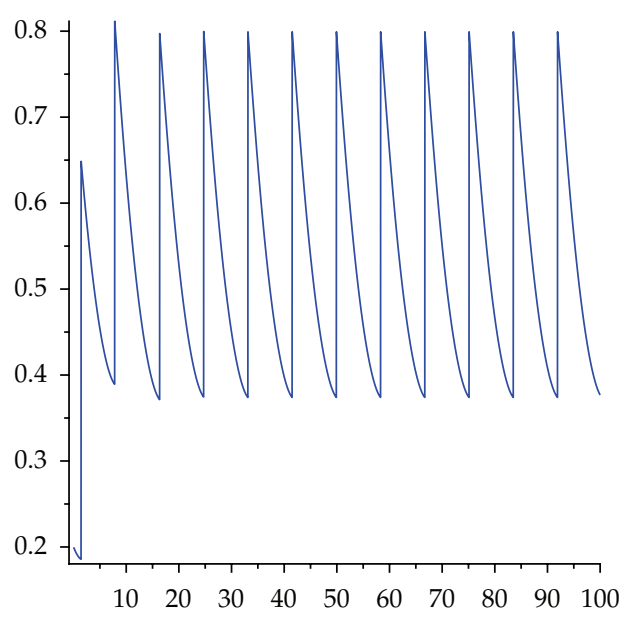

(b)

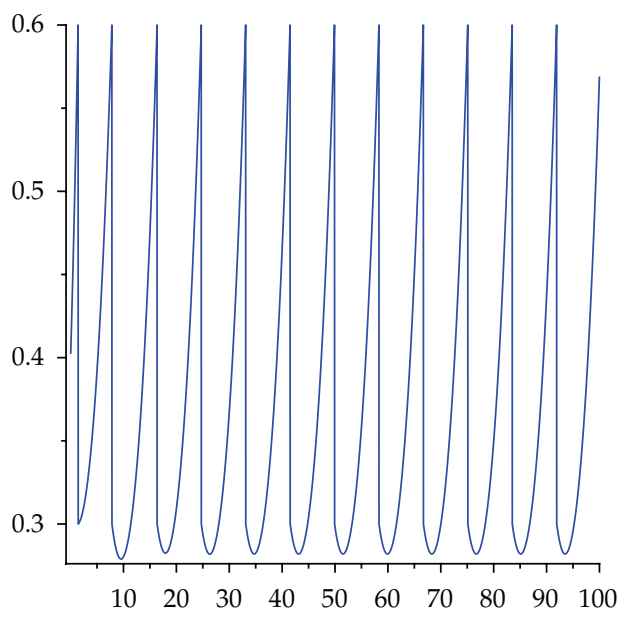

(c)

Figure 14: The time series and phase diagram for system (1.3) starting from initial value $(0.45,0.25)$ (red), $(0.4,0.2)$ (green), and $(0.3,0.1)$ (blue) $h_{1}<(1-\alpha) h_{2}<x^{*}<h_{2}<x_{0}$.

and the trajectory from different initiating must ultimately tend to be the order one periodic solution. Therefore, order one periodic solution is attractive.

Example 5.3. Existence and attractive of positive periodic solutions.

We set $h_{1}=0.3, h_{2}=0.8, h_{1}<(1-\alpha) h_{2}<x^{*}<h_{2}$, initiating points are $(0.4,0.1)$ (red), $(0.4,0.2)$ (green), and $(0.4,0.15)$ (blue) as shown in Figure 15. Therefore, the conditions of Theorem 3.3 and Theorem 4.3 hold, so system (1.3) exists order one periodic solution, and it is attractive.

Example 5.4. Existence and attractive of positive periodic solutions. 


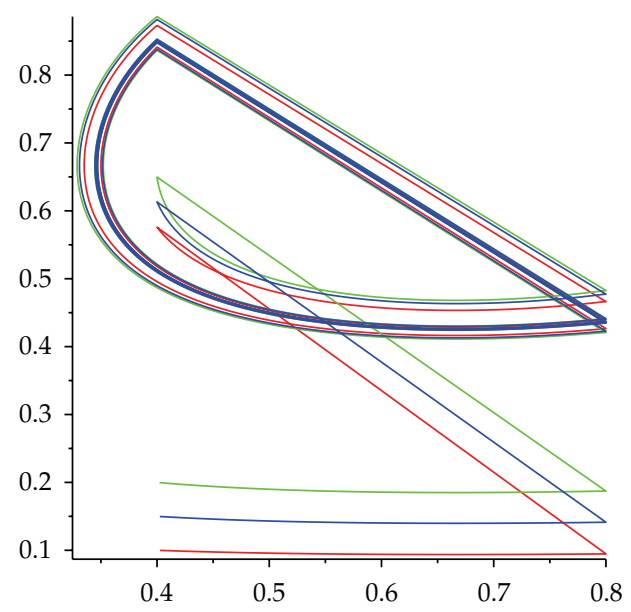

(a)

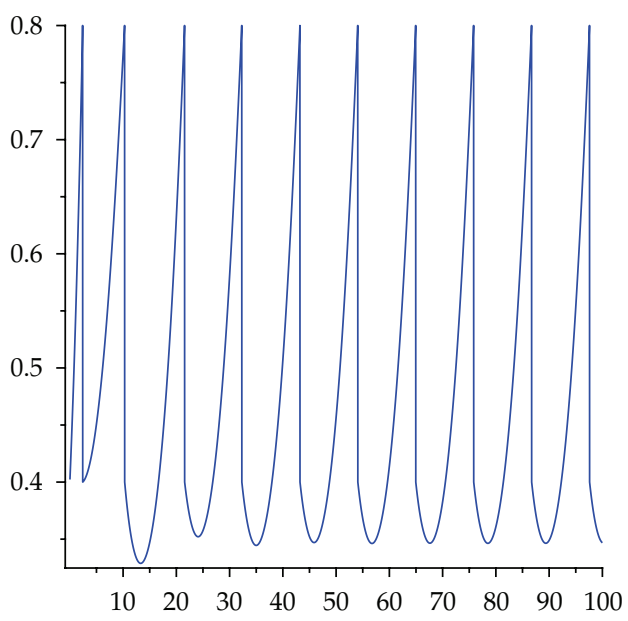

(b)

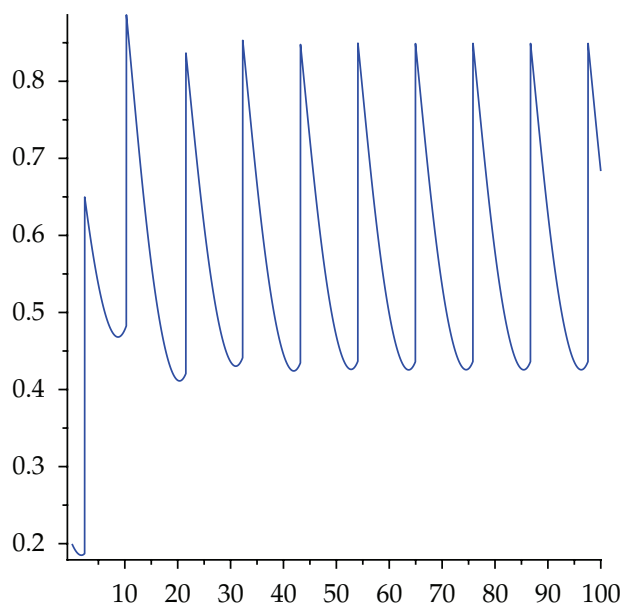

(c)

Figure 15: The time series and phase diagram for system (1.3) starting from initial value $(0.4,0.1)$ (red), $(0.4,0.2)$ (green), and $(0.4,0.15)$ (blue) $h_{1}=0.3, h_{2}=0.8, h_{1}<(1-\alpha) h_{2}<x^{*}<h_{2}$.

We set $h_{1}=0.3, h_{2}=1.5, h_{1}<(1-\alpha) h_{2}<x^{*}<x_{0}<h_{2}$, initiating points are $(0.9,0.2)$ Figure 16 shows that results of Case 4 are valid.

These results show that the state-dependent impulsive effects contribute significantly to the richness of the dynamics of the model. Our results show that, in theory, a pest can be controlled such that its population size is no larger than its ET by applying effects impulsively once, twice, or at most, a finite number of times, or according to a periodic regime. The methods of the theorems are proved to be new in this paper, and these methods are more efficient and easier to operate than the existing research methods which have been applied to the models with impulsive state feedback control [12-15], so they are deserved further promotion. 


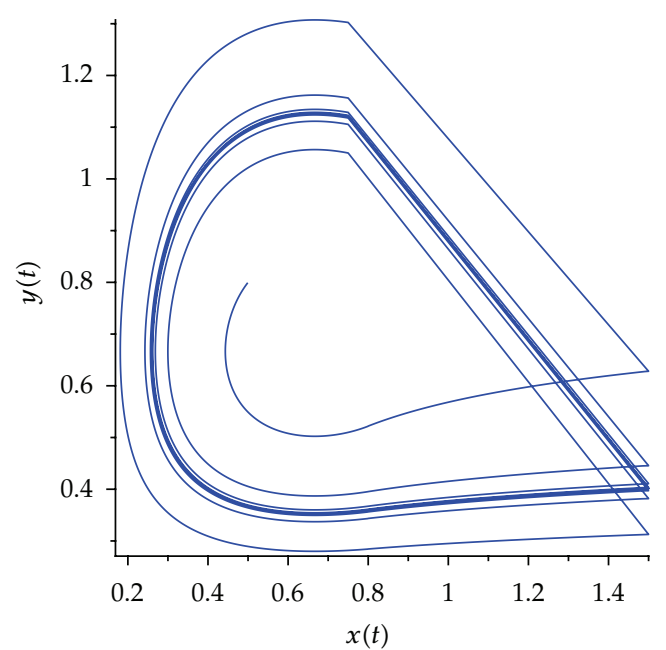

(a)

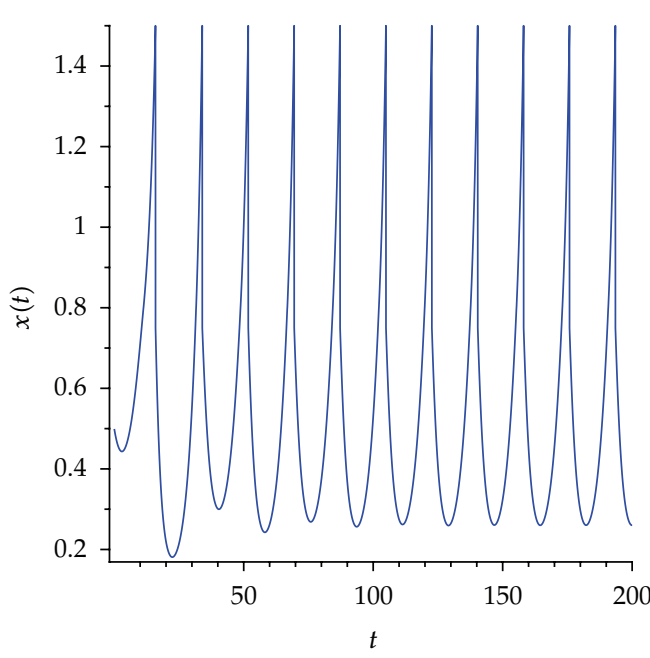

(b)

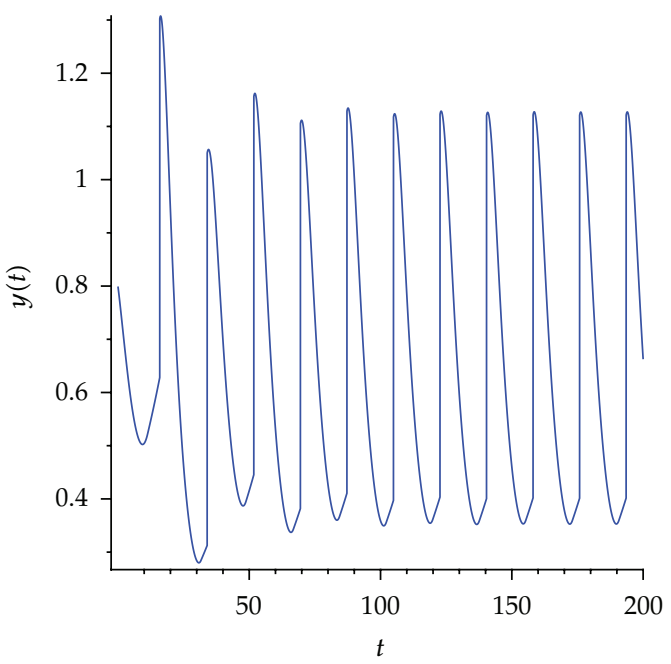

(c)

Figure 16: The time series and phase diagram for system (1.3) starting from initial value $(0.9,0.2) h_{1}=$ $0.3, h_{2}=1.5, h_{1}<(1-\alpha) h_{2}<x^{*}<x_{0}<h_{2}$.

\section{Acknowledgment}

This project was supported by the National Natural Science Foundation of China (no. 10872118).

\section{References}

[1] H. Zhang, J. Jiao, and L. Chen, "Pest management through continuous and impulsive control strategies," BioSystems, vol. 90, no. 2, pp. 350-361, 2007.

[2] X. Song, M. Hao, and X. Meng, "A stage-structured predator-prey model with disturbing pulse and time delays," Applied Mathematical Modelling, vol. 33, no. 1, pp. 211-223, 2009. 
[3] H. Zhang, L. Chen, and P. Georgescu, "Bifurcation of nontrivial periodic solutions for an impulsively controlled pest management model," Applied Mathematics and Computation, vol. 202, no. 2, pp. 675687, 2008.

[4] X. Meng and L. Chen, "A stage-structured SI eco-epidemiological model with time delay and impulsive controlling," Journal of Systems Science E Complexity, vol. 21, no. 3, pp. 427-440, 2008.

[5] L. Nie, J. Peng, Z. Teng, and L. Hu, "Existence and stability of periodic solution of a Lotka-Volterra predator-prey model with state dependent impulsive effects," Journal of Computational and Applied Mathematics, vol. 224, no. 2, pp. 544-555, 2009.

[6] S. Tang, Y. Xiao, L. Chen, and R. A. Cheke, "Integrated pest management models and their dynamical behaviour," Bulletin of Mathematical Biology, vol. 67, no. 1, pp. 115-135, 2005.

[7] G. Jiang, Q. Lu, and L. Peng, "Impulsive ecological control of a stage-structured pest management system," Mathematical Biosciences and Engineering. MBE, vol. 2, no. 2, pp. 329-344, 2005.

[8] B. Liu, Y. Zhang, and L. Chen, "Dynamic complexities of a Holling I predator-prey model concerning periodic biological and chemical control," Chaos, Solitons and Fractals, vol. 22, no. 1, pp. 123-134, 2004.

[9] G. Jiang and Q. Lu, "Impulsive state feedback control of a predator-prey model," Journal of Computational and Applied Mathematics, vol. 200, no. 1, pp. 193-207, 2007.

[10] G. Ballinger and X. Z. Liu, "Permanence of population growth models with impulsive efects," Mathematical and Computer Modelling, vol. 26, no. 12, pp. 59-72, 1997.

[11] S. Tang and R. A. Cheke, "State-dependent impulsive models of integrated pest management (IPM) strategies and their dynamic consequences," Journal of Mathematical Biology, vol. 50, no. 3, pp. 257-292, 2005.

[12] B. Liu, Y. Zhang, and L. Chen, "Dynamic complexities of a Holling I predator-prey model concerning periodic biological and chemical control," Chaos, Solitons and Fractals, vol. 22, no. 1, pp. 123-134, 2004.

[13] G. Jiang, Q. Lu, and L. Peng, "Impulsive ecological control of a stage-structured pest management system," Mathematical Bioscienceand Engineering, vol. 2, no. 2, pp. 329-344, 2005.

[14] J. Jiao and L. Chen, "Global attractivity of a stage-structure variable coefficients predator-prey system with time delay and impulsive perturbations on predators," International Journal of Biomathematics, vol. 1, no. 2, pp. 197-208, 2008.

[15] C. S. Holling, "The functional response of $P$ redator to prey density and its role in mimicry and population regulation," Memoirs of the Entomological Society of Canada, vol. 45, pp. 1-60, 1965.

[16] K. Sun, A. Kasperski, Y. Tian, and L. Chen, "Modelling and optimization of a continuous stirred tank reactor with feedback control and pulse feeding," Chemical Engineering and Processing, vol. 50, no. 7, pp. 675-686, 2011. 


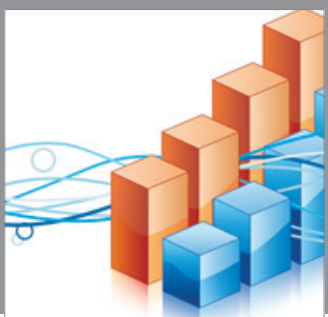

Advances in

Operations Research

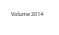

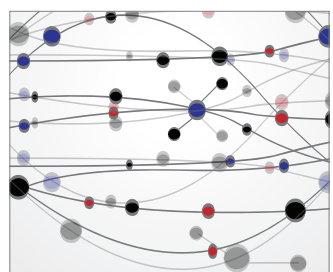

\section{The Scientific} World Journal
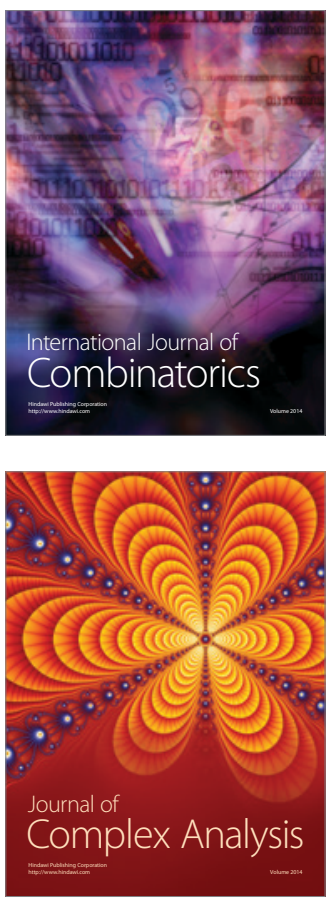

International Journal of

Mathematics and

Mathematical

Sciences
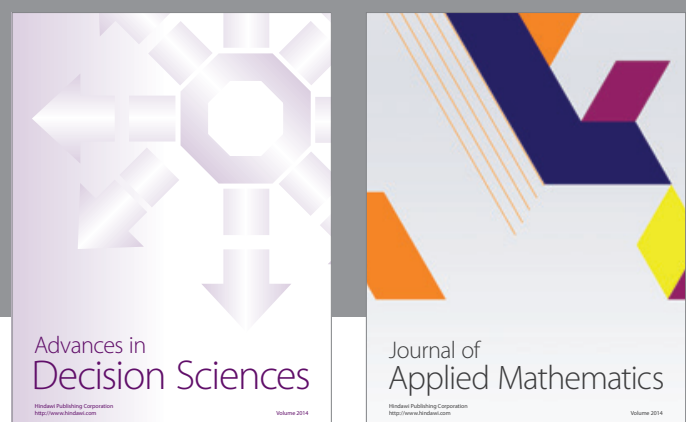

Journal of

Applied Mathematics
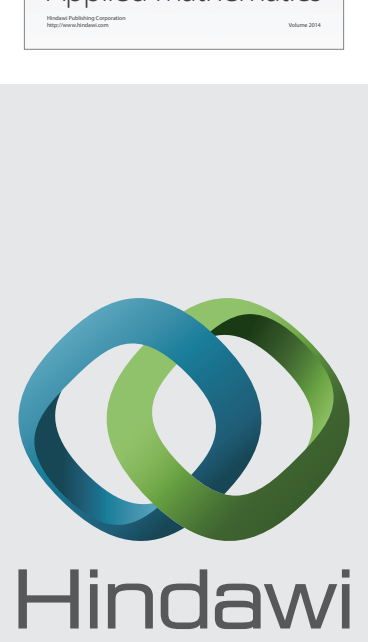

Submit your manuscripts at http://www.hindawi.com
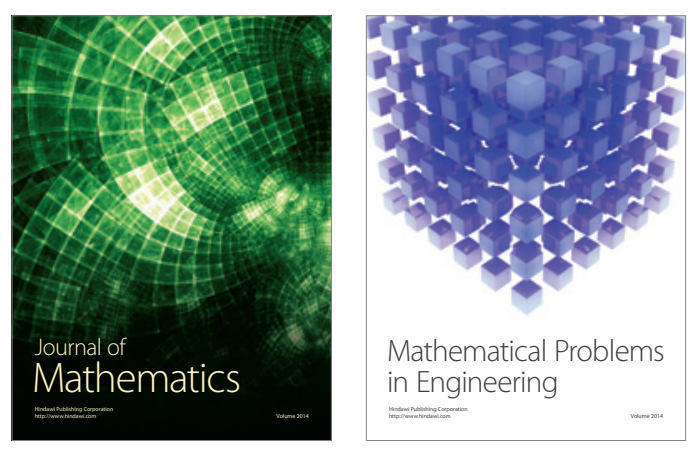

Mathematical Problems in Engineering
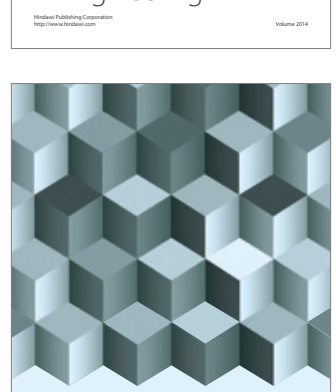

Journal of

Function Spaces
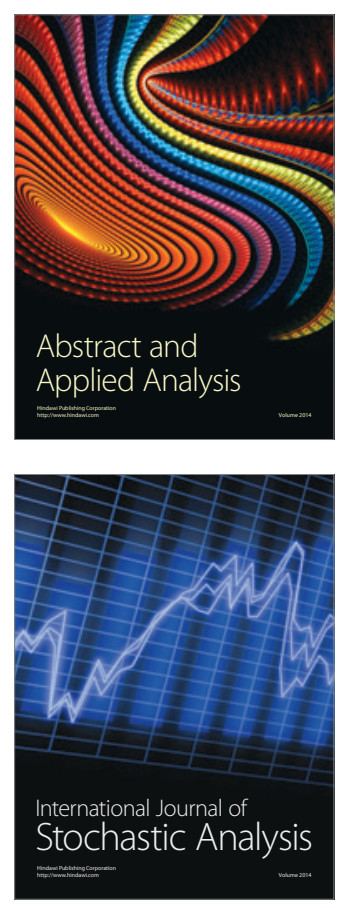

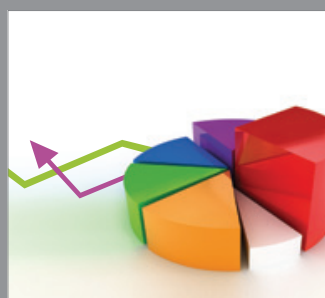

ournal of

Probability and Statistics

Promensencen
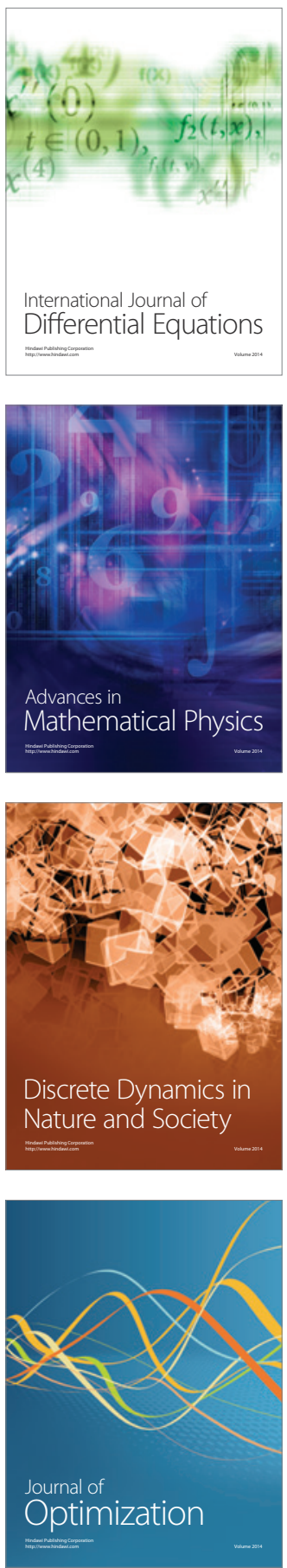